\title{
An Empirical Study and Implications on Chinese Students' Learning Related Academic Emotions
}

\author{
Xian Hua Yang ${ }^{1, a^{*}}$, Shu Yan $\mathrm{Xu}^{2, \mathrm{~b}}$ \\ ${ }^{1}$ College of Humanities and Social Sciences of Shangluo University, Shangluo of Shaanxi, China \\ ${ }^{2}$ College of Language and Culture Communications of Shangluo University, Shangluo of Shaanxi, \\ China \\ *a emery10818@sina.com, ${ }^{b}$ lorraine2004@163.com
}

Keywords: Learning emotions; Academic emotions; Chinese students; Empirical study.

\begin{abstract}
By using college students' learning-related academic emotions questionnaire, learning related academic emotions for Chinese students are explored on its current situation and its related factors. The results show that students' learning emotions consisted of eight ones, and presented mainly in positive ones; there are significant differences on emotions in gender, major and grade; family economic status has significant impacts on students' learning emotions. The implications of the research on education and teaching are discussed finally.
\end{abstract}

\section{Introduction}

In recent years, academic emotions have become one of the hot issues on the field of educational psychology, which have been aroused researchers' attention worldwidely[1]. For example, Pekrun et al(2002) explored college students' academic emotions by using qualitative and quantitative research in Educational Psychologist; In Acta Psychological Sinica, Dong Yan et al(2007) developed an effective academic emotions questionnaire based on three samples consisting of 1071 Chinese adolescents and applied it into adolescents' academic emotions. However, studies of college students' academic emotions at home start relatively late. Concerning to local universities, for the influencing factors of their special geographical position, cultural environment and academic levels ect, the contents, constructs and characteristic of academic emotions for undergraduate students in these universities may be different, and researchers at home especially should pay attention to these subjects and develop ecological study combining with their localization. Based on the retrieval and arrangement on the literature of academic emotions, this study aims to investigate the current situations, characteristics and its influencing factors for local universities students' learning emotions which are one of the most important academic emotions by empirical study, and also provide evidence for the research or practice on education and teaching of college students' academic emotions.

\section{Methods}

Participant. Effective subjects are 414 Chinese undergraduate students, including 153 boys, 261 girls; 54 only children, 360 non-only children; 107 urban students, 307 rural students; 242 students of liberal arts, 172 science students; 145 freshman, 141 sophomores, 128 juniors; 53 students' fathers' education background are under primary school, 180 junior middle school, 136 senior middle school, 24 junior college, 21 bachelor degree or above; 103 students' mothers' education background are under primary school, 194 junior middle school, 87 senior middle school, 22 junior college, 8 bachelor degree or above; 136 students' family per capita monthly income(RMB) are under 500, 168 between 500 and 1000, 73 between 1000 and 2000, 26 between 2000 and 3000, 11 above 3000 .

Measurement. The Chinese version of college students' learning-related academic emotions questionnaire (L-AEQ). The original English version questionnaire is developed by Pekrun and his members. For its good reliability and validity and its cross-cultural applicability, nowadays it has been 
widely used by many different countries. After revision, L-AEQ altogether has 60 items, including eight emotions, that is boredom, anger, enjoyment, hope, hopelessness, anxiety, pride and shame [2]. When measuring students' learning emotions, it is used by 5-point scale, and every item is scored from 1 to 5 grades from completely disagree to wholly agree. The Cronbach's a of each emotion in this study among 0.695 to 0.802 . Self-made basic data questionnaire for college students. It is mainly examine gender, major, student source, the only condition, grade, parents' education background, and family economic situation and so on.

Research Procedure, Data Analysis and Statistics Processing. Send out questionnaires with class for the unit, collect them on the spot after they are answered, then eliminate the invalid ones. And then input, tackle and analyze the valid data by SPSS 11.5 software package, the main statistics method including description statistics, independent samples group t test, and single factor variance analysis.

\section{Results}

Basic Condition of Students' Learning Emotions. Results of description statistics show that undergraduate' learning emotions scores from high to low are enjoyment $(4.01 \pm 0.54)$, hope (3.80 \pm 0.61$)$, pride $(3.70 \pm 0.61)$, anxiety $(2.88 \pm 0.68)$, boredom $(2.82 \pm 0.77)$, shame $(2.62 \pm 0.68)$, anger $(2.24 \pm 0.71)$, hopelessness $(1.89 \pm 0.69)$. This shows that undergraduates in local colleges are mainly positive emotions.

Characteristics of Students' Learning Emotions. The $t$ test on the difference of students' learning emotions, who differ in gender, major, student source and the only child condition, show that significant differences exist: ( 1 ) in different gender, students' anxiety $(\mathrm{t}=-2.58, \mathrm{P}<0.05)$, enjoyment $(\mathrm{t}=-2.68, \mathrm{P}<0.01)$, and pride $(\mathrm{t}=-2.18, \mathrm{P}<0.05)$, that is, girls' score are higher than that of boys; (2) in different major, students' boredom $(\mathrm{t}=-1.96, \mathrm{P}=0.05)$ and pride $(\mathrm{t}=2.03, \mathrm{P}<0.05)$, that is, the boredom scores of science students are higher than that of students of liberal arts, whereas the latter's pride scores are higher than that of the former; (3) in different student source, the rural students' shame $(\mathrm{t}=-2.12, \mathrm{P}<0.05)$ scores are higher than that of urban students; (4) in different only child condition, non-only child's shame $(\mathrm{t}=-2.71, \mathrm{P}<0.01)$ scores are higher than that of only child. Variance analysis of the difference of students' learning emotions, who differ in grade, shows that significant differences exist in different grades students' anxiety $(\mathrm{F}=4.35, \mathrm{P}<0.05)$, boredom $(\mathrm{F}=16.54, \mathrm{P}=0)$, enjoyment $(\mathrm{F}=3.53, \mathrm{P}<0.05)$, hope $(\mathrm{F}=7.30, \mathrm{P}=0.001)$, pride $(\mathrm{F}=3.85, \mathrm{P}<0.05)$. Results of multiple comparisons reveal that the anxiety and boredom scores of sophomores and juniors are higher than that of freshmen, while the enjoyment and hope scores of freshmen are higher than that of juniors, and the pride scores of sophomores are higher than that of juniors. Therefore from the above we can conclude that girls experience more positive and negative emotions, science students, rural students and non-only child experience more negative emotions, and lower grade students experience more positive emotions and less negative ones.

Influencing Factors on Students' Learning Emotions. Results of variance analysis reveal that parents' education background has no remarkable influence on students' learning emotions $(\mathrm{F}<1.98, \mathrm{P}>0.05)$, while family financial situation greatly influences students' shame emotion $(\mathrm{F}=3.47, \mathrm{P}<0.01)$. To be specific, the shame scores of students, whose family per capita income is less than one thousand RMB, are higher than that of students whose family per capita income is between one thousand and two thousand RMB. This shows that although parents' education background has no significant effect on students' learning emotions, their family financial situation is an important factor to influence it.

\section{Discussions}

Outcomes of empirical study. This study shows that Chinese college students' learning emotions mainly consist of 8 kinds of emotions, which are consistent with most previous researches. Ma Huixia 
and her group find[3] that average scores of college students' learning emotions from high to low are hope, enjoyment, pride, relax, interest, shame, anger, anxiety, boredom, and hopelessness; Yang Xianhua also find[4] that the average scores of middle school students' classroom mathematics learning emotions from high to low are pride, enjoyment, anxiety, boredom, shame and hopelessness. But at present we cannot conclude that the domestic students' learning emotions are mainly positive ones without the support from further study results. For example, the study from Yang Xianhua reveals that mathematics learning emotions intensity of senior high school students from strong to weak are anxiety, pride, shame, enjoyment and anger.Based on the comparison of gender difference, the study gets more complex results, that is, girl's positive emotions and negative emotion are significantly higher than that of boy, which are consistent with foreign research. Eroğlu and his members find[5] that in Turkey college female students' positive learning emotions and negative emotions are significantly higher than that of male students; the research of college students' learning emotions from Su Shijiang and his members holds[6] that girl students experience more negative emotions such as anxiety, shame, anger; the investigation from Yang Xianhua on senior 2 students' classroom mathematics learning emotions suggests that girls' negative emotion is significantly higher than that of boys. However, some studies also draw the results of no gender difference. For example, the study results from Frenzel and his members show [7] that no significant difference exists in pupils' learning emotions. And Sun Shimei also find[8] that there is no significant gender difference in the six kinds of adolescents emotions, namely disgust, fear, sadness, enjoyment, anxiety and surprise. The results of this study also show that sophomores and juniors have significantly higher negative emotions than that of freshmen, and freshmen and sophomores have significantly higher positive emotions than that of juniors. This suggests that lower grade undergraduates experience more positive emotions and less negative ones, whereas the senior and junior the opposite. Eroğlu and his members also find that higher grade college students experience more anxiety and hopelessness than that of lower grade college students, while the lower grade college students experience more hope than that of higher grade college students. The researchers at home get similar results from the study on middle school students' learning emotions. For instance, Dong Yan et cl find[9] that junior middle school students have more positive emotions and less negative emotions than senior middle school students; the study from Yang Xianhua[10] shows that senior middle school students have higher scores in boredom, hopelessness, anxiety, and shame than junior middle school students, while junior middle school students have higher scores in enjoyment and pride the two positive emotions than senior middle school students; Sun Shimei also find that senior middle school students experience higher sadness emotion than junior middle school students. This study find family financial situation greatly influences students' shame emotion, that is, students whose family per capita income is less than one thousand, are higher than that of students whose family per capita income is between one thousand and two thousand. This reveals that family financial situation is an important factor to influence college students' learning emotions, however, the specific influencing mechanism needs further research.

Enlightenments of empirical study. The enlightenments of empirical study of learning emotion on education and teaching are as fowolls. The study has some enlightenment on Chinese college students' education teaching research and practice: In research, researchers should strengthen the theory research, integrating "scattered" empirical results. Because of the differences in measuring tool, study object, ecological environment, cultural background, and the current studies at home and abroad in the field of learning emotions differ greatly and show some degree of scatter. In practice, teachers in the education teaching should strengthen emotional class design, excavate students' positive emotional force, and promote the development of the students' learning emotions. On the basis of characteristics of different gender, majors etc students and their family influencing factors, teachers should teach and encourage science students, rural students, the non-only child and lower family financial situation students construct positive attribution and high subjective value sense, in order to reduce the effect of negative emotions and improve the education quality of teaching; in addition, students themselves should study more scientific knowledge about learning emotions after 
class, learn to timely perceive and adjust their learning emotions, constantly improve their own emotional intelligence, and enhance the ability of independent learning.

\section{Conclusion}

It can be conclude as follows in this study. Chinese students' learning emotions consisted of eight ones, which from high to low are enjoyment, hope, pride, anxiety, boredom, shame, anger and hopelessness, and students' experience mainly positive emotions. There are significant differences on emotions in gender, major, grade et al. Family economic status has significant impacts on students' learning emotions that are family financial situation greatly influences students' shame. This study on students' learning emotions has many implications both on theories and practice of education and teaching.

\section{Acknowledgment}

This study was funded by the Scientific Research Program of Shangluo University (13SKY020), Shaanxi Province Education Science and the Twelfth Five Year Plan Project (SGH13402) and the Fundamental Research Funds for Students' affairs Research Branch of China Higher Education Association (LX2014Y053).

\section{References}

[1] Guoliang Yu and Yan Dong, Study of Academic Emotion and Its Significance for Students' Development. Educational Research, pp.193-197, 2009(In Chinese).

[2] Xianhua Yang, Preliminary Revision of Chinese Version of Learning-related Academic Emotions Questionnaire for College Students. China Journal of Health Psychology, in press, 2011(In Chinese).

[3]Huixia Ma, Development of the General Academic Emotion Questionnaire for College Students. Chinese Journal of Clinical Psychology, pp. 593-596, 2008(In Chinese).

[4]Xianhua Yang, On Problem Analysis of Mathematics Achievement Emotions under the Background of New Curriculum Reform. Journal of Shangluo University, pp. 77-80, 2009(In Chinese).

[5]Eroğlu S E, Işiklar A and Bozgeyikli H, Research of university students' academic emotions regarding some variables: selÇuk university faculty of education sample. Georgian Electronic Scientific Journal: Education Science and Psychology, pp. 33-42,2006.

[6]Shijiang Su and Huixia Ma, Research on General Academic Emotion of College Students and It's Relationship with Adjustment. China Journal of Health Psychology, pp. 591-593, 2009(In Chinese).

[7]Frenzel A C, Thrash T M, Pekrun R, et al, A cross-cultural comparison of German and Chinese emotions in the achievement context. Journal of Cross-cultural Psychology, pp.302-309,2007.

[8]Shimei Sun, The Developmental Characteristics and Correlation with Self-regulated Learning of Academic Emotion for Adolescent. Master Degree Thesis of Shandong Normal University, pp. 25-29, 2006(In Chinese).

[9]Yan Dong and Guoliang Yu, The Development and Application of An Academic Emotions Questionnaire. Acta Psychologica Sinica, pp. 852-860, 2007(In Chinese).

[10]XianhuaYang, Features of High School Students' Achievement Emotionsand Their Relations with Achievement Goals. Shandong Normal University Master Thesis, pp. 1-8, 2008(In Chinese). 